\section{Agricultural genetics}

\section{Moving in on plant genes}

\author{
Robert Shields
}

VirTually nothing is known about the protein products of the genes controlling morphology, dormancy, photoperiodicity and disease resistance in plants. Two techniques being used to try to clone these genes are transposon tagging and gene mapping. Transposons (mobile pieces of DNA) can generate different phenotypes which should allow the genes governing the phenotype to be isolated; and mapping should allow specific genes to be targeted. Although tagging is the more elegant technique, a new report ${ }^{1}$ by Young et al. indicates that mapping may be the first method by which one of these genes is uncovered.

Transposons can be used as simultaneous mutagens and gene tags. Several plant genes (mainly those involved in pigment biosynthesis) have been cloned in this way from maize or snapdragon. The discovery $^{2}$ that the transposon Ac in maize could be transferred to other plants, where it is moved from its original site of insertion to a new locus, was therefore met with considerable excitement. Selffertilization of the resulting plant will give the tagged locus in a homozygous state and simultaneously reveal a new phenotype. But things may not be that straightforward. In its native host, Ac tends to excise and then reinsert at a closely linked site on the same chromosome $e^{3}$. If this is true in the heterologous host, it will be necessary to produce plant lines with transposons inserted close to target genes before tagging can begin. In other organisms such as the fruitfly Drosophila, where transposons act as mutagens, specific loci are mutated at vastly different rates as the elements show strong site-specificity for insertion ${ }^{4}$. So, although it may be possible to isolate plant genes by transposon tagging, there may be problems in approaching specific loci by this method.

In several plant species, genes have

\section{0 years ago}

WITH reference to A. C. Haddon's account of the use of the Remora or sucker fish by the natives of Torres Straits in fishing for turtles (Nature, 17 January, p.285, 1889), I may call attention to the paper on this subject read by Frederick Holmwood, C. B., late H.B.M. Consul at Zanzibar, before the Zoological Society of London, on 17 June 1881, which Haddon does not seem to be acquainted with. Holmwood has fully described the mode of the use of the Remora by the native fisherman of Zanzibar in catching turtles and fishes. It is curious to find a somewhat similar method of employing the Remora practised by the islanders of Torres Straits. P. L. SClater From Nature 39, 295; 24 January 1889. been assigned to positions in genetic linkage groups which in a few plants can be correlated with cytologically identifiable chromosomes. The resolution of many of these linkage maps is poor and several laboratories are attempting to improve them by constructing maps based on restriction fragment length polymorphisms (RFLPs). These maps have a use as a practical breeding aid, for if an RFLP marker shows close linkage with a desirable gene then progeny in breeding experiments can be rapidly screened for its presence without the necessity for large-scale field trials. A secondary use of such maps is to use them as starting points for attempts to isolate the linked gene.

The first requirement is to position the target gene accurately on the RFLP

and the RFLP map is limited by the frequency of allelic variation in the organism examined (which determines the likelihood of finding RFLPs) and the number of cases that can be examined where the target gene and closely linked RFLPs are segregating (which gives the map position). In practical terms 1 centimorgan (1 per cent recombination) is close to the limit of resolution achievable without examining a prohibitively large number of crosses unless near-isogenic lines can be used to give closer linkage. The table shows that for plants such as Arabidopsis, which has a very small genome, 1 centimorgan represents 139 kilobases, thus chromosome walking from an RFLP marker to a genetic loci is just within the bounds of possibility, given a lot of effort. Doubtless other genes will be isolated in this way from Arabidopsis and will then be used as probes to find homologous sequences in other plants. But it seems unlikely that genes governing many complicated plant functions (such as disease resistance) would be similar

The resolution gap in plants

\begin{tabular}{lcccc}
\hline Species & $\begin{array}{c}\text { Genome size } \\
\text { (kilobase pairs) }\end{array}$ & $\begin{array}{c}\text { Map length } \\
\text { (centimorgan) }\end{array}$ & $\begin{array}{c}\text { Kilobase pairs equivalent } \\
\text { to 1 centimorgan }\end{array}$ & Ref. \\
Arabidopsis & $7 \times 10^{4}$ & 501 & 139 & 10 \\
Tomato & $7.15 \times 10^{5}$ & 1,400 & 510 & 1 \\
Human & $3 \times 10^{6}$ & 2,708 & 1,108 & 11 \\
Maize & $3 \times 10^{6}$ & $\sim 1,400$ & $\sim 2,140$ & 12
\end{tabular}

Map lengths are based on published RFLP data. The conversion of centimorgans to kilobase pairs is an average value as recombination frequency varies in different chromosome regions.

linkage map and if necessary find new RFLPs which map closer to the gene. Young et al. have now elegantly demonstrated ${ }^{1}$ the combined use of RFLPs and near-isogenic lines to find markers very close to a plant disease-resistance gene. Plant breeders often cross a cultivated variety with an alien species carrying a desired gene and then backcross to the cultivated variety (the recurrent parent) while selecting to maintain the required trait. After a series of such crosses, the genome of the selected plants will consist almost entirely of the genome of the recurrent parent with a small segment surrounding the target gene which comes from the alien genome. By examining RFLP differences between the original variety and the new near-isogenic line, it is possible to identify RFLP markers lying very close to the introgressed gene. Young et al. find markers lying $0.4 \pm 0.4$ centimorgans $\left(=2 \times 10^{5}\right.$ base pairs in tomato) of the $\operatorname{Tm} 2 a$ gene (a dominant gene giving resistance to tobacco mosaic virus by restricting cell-to-cell spread) which had been introgressed into the cultivated tomato (Lycopersicon esculentum) from the wild variety $L$. peruvianum. Near-isogenic lines should be extremely valuable for rapidly assigning introgressed genes to their map position.

The correlation between the genetic between Arabidopsis and economically important crop plants.

For such plants a 'resolution gap' exists between the genetic and the physical map which must be bridged if their genes are to be approached from linked RFLP probes. A similar (although less daunting; see table) resolution gap in the human genome has spurred technical developments such as pulsed-field gel electrophoresis ${ }^{5}$ and cloning in yeast artificial chromosomes to handle and map very large pieces of DNA $^{6.7}$ as well as the use of 'jumping' and 'linking' libraries to overcome the necessity of walking everywhere in the genome ${ }^{8.9}$. Once the region surrounding the target gene is physically mapped, ways must be found to identify the gene itself.

1. Young, N.D. et al. Genetics 120, 579-585 (1988).

2. Baker, B. et al. Proc. natn. Acad. Sci. U.S.A. 83, 4844 4848 (1986)

3. Greenblat, I.M. Genetics 108, 471-485 (1984).

4. Cooley, L. et al. Science 239, 1121-1128 (1988).

Schwartz, D.C. et al. Cell 37, 67-75 (1984)

6. Burke D.T Science 236, 806-812 (1987)

7. Vollrath, D et al. Proc, natn. Acad Sci. US.A. 85, 6027 6031 (1988)

8. Richards, J.R.E. et al. Proc. natn. Acad. Sci. U.S.A. 85, 6437-6441 (1988).

9. Pohl, T.M. et al. Nucleic Acids Res. 16, 9185-9198 (1988).

10. Chang, C. et al. Proc. natn. Acad. Sci. U.S.A. 85, 6856$6860(1988)$

1. Donnis-Keller, H. et al. Cell 51, 319-337 (1987) 12. Helentjaris, T. et al. Genetics 118, 353-363 (1988).

Robert Shields is at Plant Breeding International, Trumpington, Cambridge $C B 22 L Q, U K$. 